\title{
Diaphragmatic Paralysis
}

\author{
Swetha Gadwala MD, Gilbert Berdine MD
}

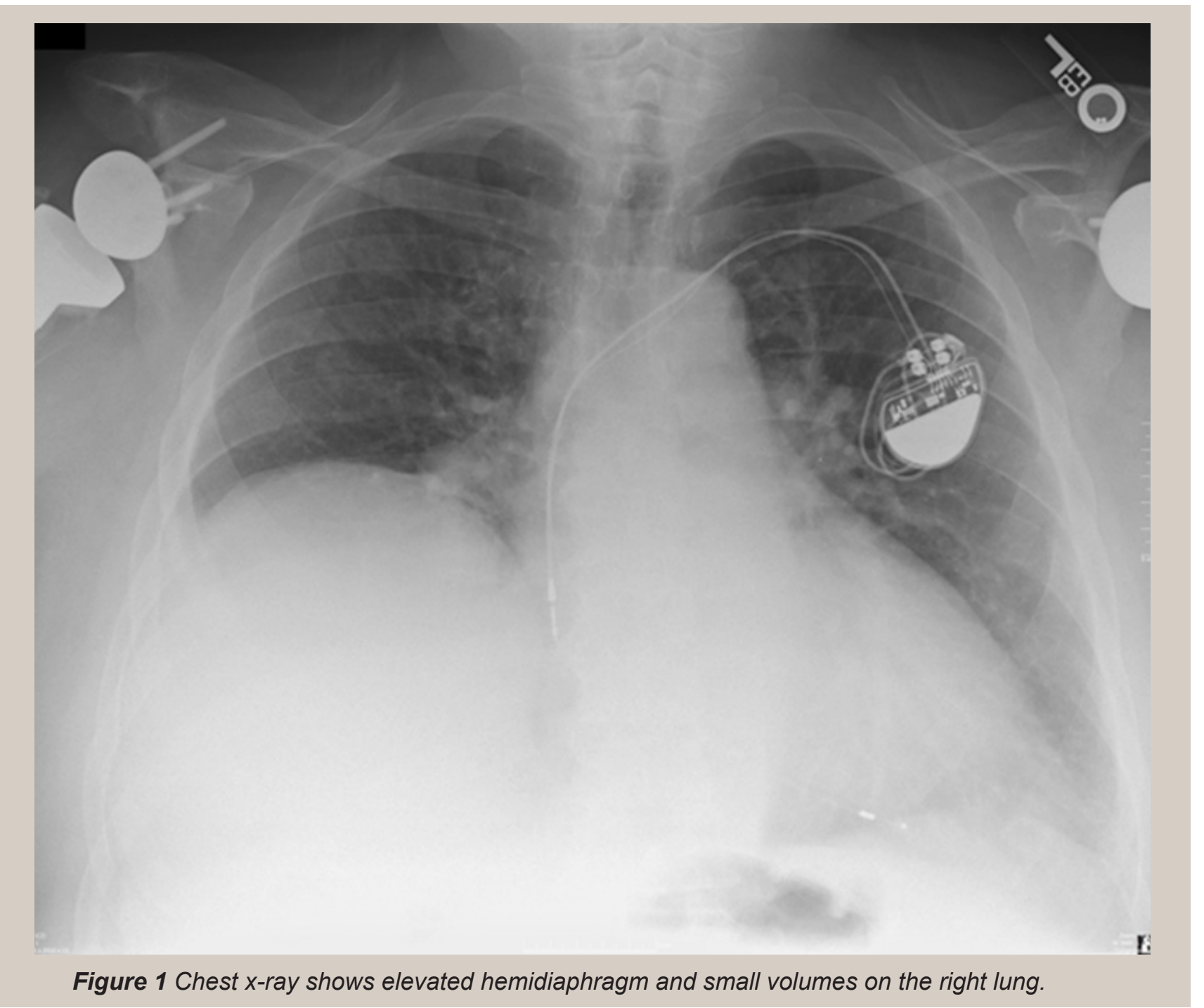

\section{INTRODUCTION}

The diaphragm is a musculofibrous, domeshaped structure attached to the first, second, and

Corresponding author: Swetha Gadwala MD

Contact Information: Swetha.gadwala@ttuhsc.edu

DOI: 10.12746/swrccc 2013.0104.043 third lumbar vertebrae posteriorly, to the lower sternum anteriorly, and to the costal arches laterally. It consists of sternal, costal, and lumbar muscle groups and a large fibrinous central tendon. The major blood supply comes from the left and right phrenic arteries. It is exclusively innervated by the right and left phrenic nerves, originating from C3-4-5. 


\section{Causes}

Diaphragm weakness could be due to congenital defects (Hernia of Morgagni, Hernia of Bochdalek), acquired defects (acute or chronic diaphragmatic hernias), eventration (muscle weakness), paralysis (direct injury to diaphragm or phrenic nerve), reduced diaphragmatic pacing (central alveolar hypoventilation or high cervical cord injuries), and tumors. The most frequent causes are accidents, birth trauma, and postoperative complications of cardiovascular surgery. Unilateral paralysis is due primarily to tumors infiltrating the phrenic nerve. It can occasionally occur due to a complication of neurologic disease or be idiopathic.

\section{SYMPTOMS}

The symptoms depend on stage of paralysis, acute or chronic, bilateral or unilateral, and on pre-existing conditions. It causes decreased vital capacity at rest, especially in supine positions. The greater load and gas exchange impairment leads to hypoxemia at rest, during sleep, and with exercise. Orthopnea is immediate upon lying down, compared to the delayed orthopnea in patients with heart failure. Other symptoms include dyspnea on bending and carrying light weights, abdominal pain due to excessive load on abdominal muscle, and respiratory insufficiency with severe hypoxia and $\mathrm{CO} 2$ retention. Acute onset paralysis presents with acute distress, severe orthopnea, shoulder pain, and fatigue imitating cardiovascular process, thereby delaying diagnosis.

\section{DIAGNOSIS}

History and physical examination should focus on paradoxical movement of the abdominal wall. A plain chest $\mathrm{x}$-ray showing asymmetry of the hemidiaphragm is usually the first indication of injury. Linear shadows or patchy atelectasis above the diaphragm are seen. A computed tomography can detect small tears, and an ultrasound can detect free intraperitoneal blood, especially in trauma. MRI is reserved for patients who are hemodynamically stable and cannot undergo other diagnostic imaging. Fluoroscopy (sniff test) is used to diagnose abnormal diaphragmatic motion due to phrenic nerve injury. Complete paralysis will be visualized as absent or paradoxical motion of the affected side.

\section{TREATMENT}

Treatment depends on severity and the patient's baseline status. Prognosis for unilateral paralysis in patients without underlying pulmonary disease is generally good and rarely requires treatment. Respiratory pacemakers can be used for those with functioning phrenic nerves. In bilateral diaphragm injury, continuous positive airway pressure or mechanical ventilation and tracheostomy are needed for survival. Prognosis remains poor in patients with bilateral paralysis, advanced lung disease, and chronic demyelinating conditions.

Received: $8 / 19 / 2013$

Accepted: 8/29/2013

Reviewers: Kenneth Nugent MD

Published electronically: 10/16/2013

Conflict of Interest Disclosures: None

\section{References}

1. Ben-Dov, I. Diaphragmatic Paralysis- Symptoms, Evaluation, Therapy and Outcome. (http://cdn.intechopen.com/ pdfs/37827/InTech-Diaphragmatic paralysis symptoms evaluation therapy and outcome.pdf

2.Levitzky, M.G., 2013. Chapter 2. Mechanics of Breathing, in Pulmonary Physiology, 8th edition, McGraw-Hill

3. Nason, L.K. et al. Radiographics. 2012 Mar-Apr; 32(2); E51-70.doi: 10.1148/rg.32115127. PMID 22411950 